\title{
Fabrication of Palladium Nanoparticles by Laser Ablation in Liquid
}

\author{
T. Nishi, A. Takeichi, H. Azuma, N. Suzuki, T. Hioki, T. Motohiro \\ Toyota Central Research and Development Laboratories Incorporation, Nagakute, Aichi 480-1192, Japan \\ E-mail:e1481@mosk.tytlabs.co.jp
}

\begin{abstract}
Laser ablation of a palladium disc target immersed in heavy water or light water using second harmonic Nd:YAG laser was carried out to fabricate palladium nanoparticles. The properties of palladium nanoparticles ablated in heavy water or light water were investigated and compared. It is shown that liquid with smaller optical absorption coefficient improve the yield of nanoparticles. The transmission electron microscopy observation revealed that palladium nanoparticles with a diameter range from a few $\mathrm{nm}$ to several tens of nanometer were produced in light water while the average diameter of palladium nanoparticles produced in heavy water was smaller. Magnetic properties of palladium nanoparticles were also measured. M-H curves show palladium nanoparticles were superparamagnetic. Furthermore, laser ablation in heavy water or light water causes larger magnetic susceptibility than commercial palladium powder and plate.
\end{abstract}

DOI: $10.2961 / \mathrm{jlmn} .2010 .03 .0002$

Keyword : Palladium nanoparticle, laser ablation, magnetic susceptibility

\section{Introduction}

Recently, nanomaterials have attracted a great interest because of the unique characteristics different from bulk materials. Therefore, a wide range of synthetic approaches regarding the preparation of metal nanoparticles in various matrices, including reduction method, sol-gel process, solvent evaporation of hydrophobic colloids have been reported [1-3]. Laser ablation which is usually applied to in-situ elemental analysis [4], forming thin film (PLD: pulsed laser deposition) [5] has been also used to prepare nanoparticles [6-13]. In particular, the temperature and pressure of the plume induced by pulsed laser irradiation onto the metal target surface in liquid are very high [14, 15] compared to in vacuum or atmosphere because of confinement effect. Because there are ablated particles in this plume, crystalline nanoparticles can be obtained with no heat treatments [6]. Other advantages of laser ablation as a method to produce nanoparicles are that aggregation and dispersion can be controlled with surfactant or any adequate liquid [7-9], pure nanoparticles can be obtained, all nanoparticles can be captured in liquid. As ablated particles go through the plume induced by laser ablation in liquid in which the temperature and pressure are very high, nanoparticles with new optical, electrical and mechanical properties can be expected to be fabricated. However, the smaller yield of nanoparticles prepared by laser ablation in liquid than other method to synthesize nanoparticles is serious problem. Sasaki et al. reported the maximum ablation rate of platinum target in water was $4.4 \mathrm{mg} / \mathrm{h} \mathrm{[10]}$. The presence of nanoparticles produced by laser ablation in various liquid prevents ablation of target because ablated particles absorb or scatter the irradiation laser [11]. As the results of these effects, pulse energy reaching the target surface is smaller than that just after operating. One of the methods to resolve this problem is to use appropriate liquid with smaller absorption coefficient to prevent absorption of irradiation laser light by used liquid. However, liquid should be carefully selected because it has influence on the properties of ablated particles [7].

Palladium is one of the most interested functional materials because of a large variety of applications to hydrogen storage, fuel cell and catalyst. Nano-size crystallizing of palladium is expected to improve hydrogen storage capacity, catalyst characteristic, and magnetic property. Although bulk palladium is paramagnetic, nano-size palladium with ferromagnetic property has been theoretically predicted [16] and experimentally reported [17]. Okada et. al. [13] and Hwang et. al. [9] reported laser ablation of palladium in pure water without any surfactant and in ethyl acetate with or without tetraoctylammonium bromide $\left(\mathrm{TC}_{8} \mathrm{ABr}\right)$, respectively. Hwang employed $\mathrm{TC}_{8} \mathrm{ABr}$ to stabilize palladium nanoparticles ablated in liquid. However, magnetic properties of palladium nanoparticles prepared by laser ablation of bulk target immerged in liquid has never been reported.

In this study, laser ablation of palladium disc target immerged in heavy water or light water were carried out. The properties of palladium nanoparticles produced by laser ablation in heavy water or light water were investigated and compared.

\section{Experiments}

Fig.1 shows the schematic diagram of experimental setup for laser ablation of palladium disc target immerged in heavy water or light water. Palladium disc target was fixed on the plate connected to motor to rotate the target to prevent laser irradiation on the same spot. Second harmonic light from Nd:YAG laser system (Spectra Physics Pro-290) operated in the Q-switch mode at the wavelength of $532 \mathrm{~nm}$ with a repetition rate of $10 \mathrm{~Hz}$ and pulse duration of $7 \mathrm{~ns}$ was used. Irradiation laser light was focused on the palladium disc target by two quartz lens 


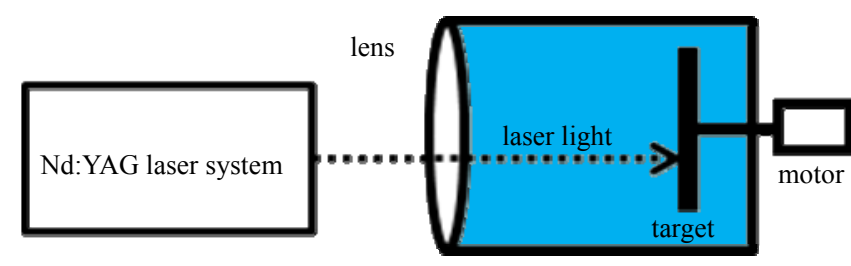

Fig.1 Schematic diagram of experimental setup for laser ablation of palladium disc target immerged in heavy water or light water.

pair which focal lengths were 50 and $70 \mathrm{~mm}$. The pulsed laser energy just after laser system was $400 \mathrm{~mJ}$. The irradiation laser diameter focused on the palladium disc target immerged in heavy water or light water with the optical length of $80 \mathrm{~mm}$ was $3.0 \mathrm{~mm}$ in diameter. Although it was $9 \mathrm{~mm}$ in diameter just after laser system, the focused size on the palladium disc target was controlled by target position in heavy water or light water. The fluence on the palladium disc target immerged in heavy water or light water were $5.66 \mathrm{~J} / \mathrm{cm}^{2}$. Laser irradiation for 1 hour was carried out four times in each case. The optical absorption by palladium nanoparticles obtained by laser irradiation onto the target immerged in heavy water or light water for 10 min were measured by spectral photometer (Shimadzu UV-2100PC) with optical length of $10 \mathrm{~mm}$. Laser ablation of palladium disc target immerged in heavy water or light water for 60 minutes were carried out to obtain palladium nanoparticles. Shapes and sizes of palladium nanoparticles were observed by transmission electron microscopy (TEM). Palladium colloids were heated up to evaporate heavy water or light water to obtain palladium nanoparticles. Magnetic properties of these samples were measured by Superconducting Quantum Interference Device (SQUID).

\section{Results and discussion}

\section{3-1 Optical absorption and ablation mechanism}

Fig. 2 shows optical absorption spectra of heavy water and light water. In the visible region, absorbance by light water

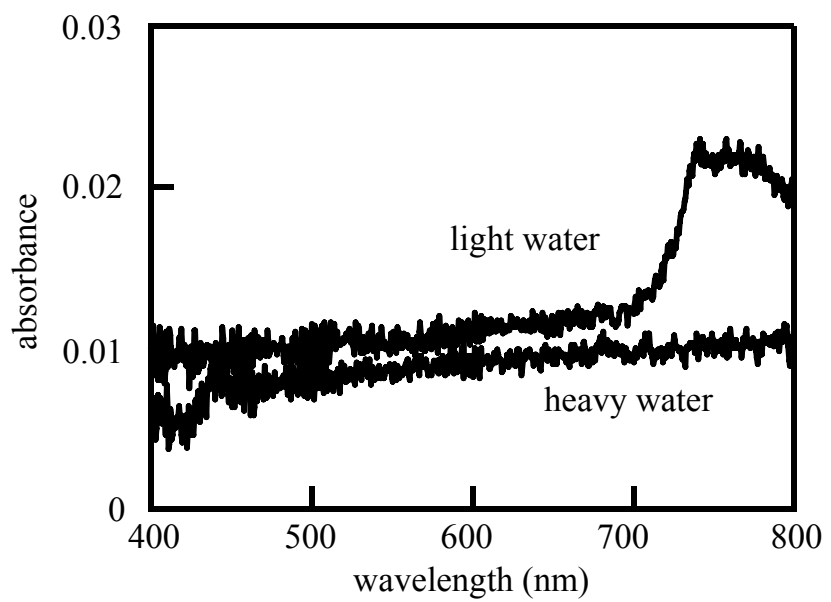

Fig.2 Optical absorption of heavy water or light water measured using optical cell with optical length of $10 \mathrm{~mm}$. is larger than that by heavy water. Therefore, pulsed laser energy on the target surface in heavy water is larger than that in light water just after starting laser irradiation even if the energies just after laser system are same. This effect is expected to result in the difference of the particle properties such as particle size and ablation rate. For long time laser ablation, we expect three processing stages. On the first stage, different fluence on the target surface contributes to the differences of ablation rate and particle size distributions. However, the first stage can't continue for more than a few minutes because laser energy reaching onto the target surface is decreased by the shielding effect of ablated particles. Therefore, it can be expected that greater shielding effect of particles ablated in heavy water than light water in the second stage due to higher laser energy on the target in the first stage. In fact, optical absorption spectra of palladium colloid prepared by laser ablation for 10 minutes in heavy water was larger than that in light water as shown in Fig.3. However, almost all energies can be absorbed by colloid prepared by previous irradiation in each case. In this stage, ablation sound couldn't be confirmed. As Sasaki reported [10], ablation yield can barely change as the function of laser fluence around ablation threshold. Therefore, ablation rate in this stage is expected to be almost same. There is expected to be a regime between first stage and second one when the absorption of colloids in each case is same. However, the change of absorption spectra in each case should be same if the regime is real. Nonetheless, absorbance of colloid in each case is quite different as shown in Fig.3. This result may indicate that the regime is not real. This may be explained by the discontinuous change of the density of palladium nanoparticles. Although ablation rate is very small, ablated particles discontinuously increase. In addition, ablation stops in less than 10 minutes as described above. As the result of it, absorbance increases during for only a few minutes. Therefore, pulsed laser irradiation to the target in colloid just below the saturation density may cause the over saturation in the case of laser ablation in heavy water. On the other hand, nanoparticle density in the case of laser ablation in light water may not exceed as same as that in heavy water because of the

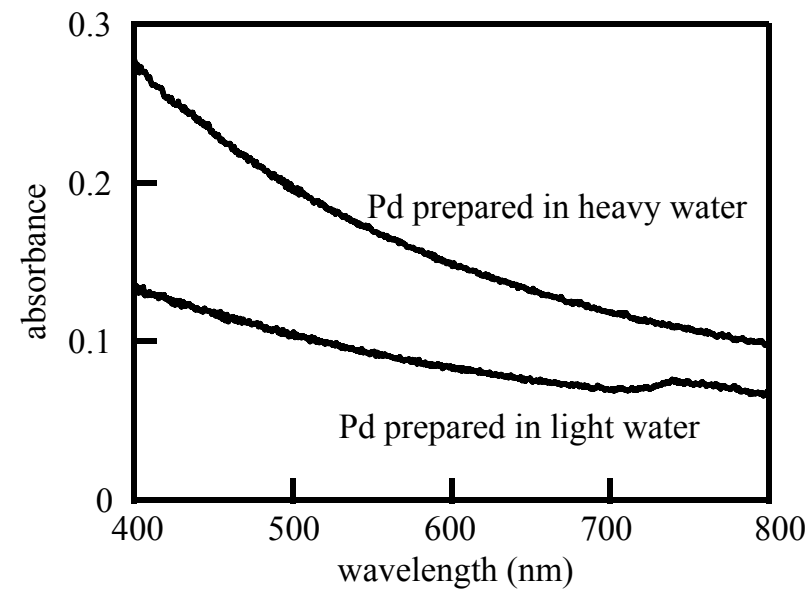

Fig.3 Optical absorption spectra of palladium colloid prepared by laser ablation in heavy water or light water. 


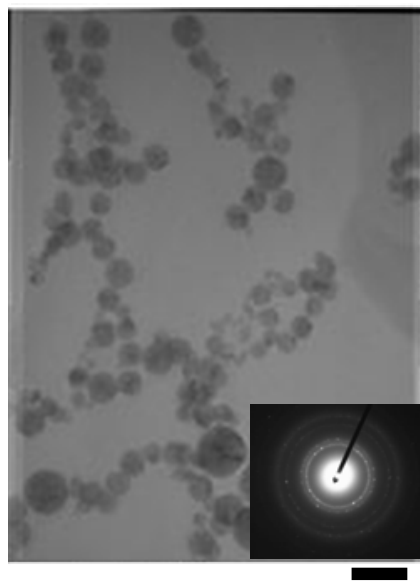

$20 \mathrm{~nm}$

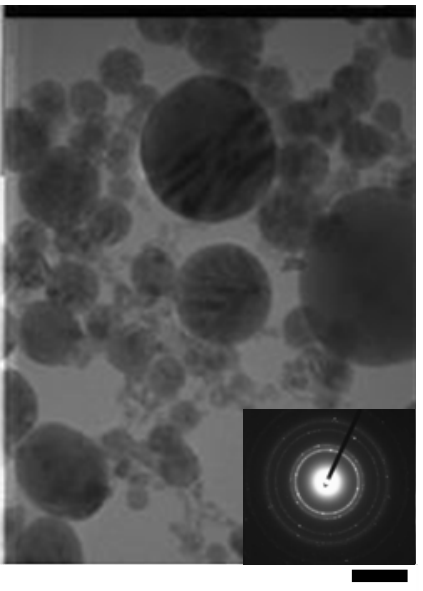

$20 \mathrm{~nm}$
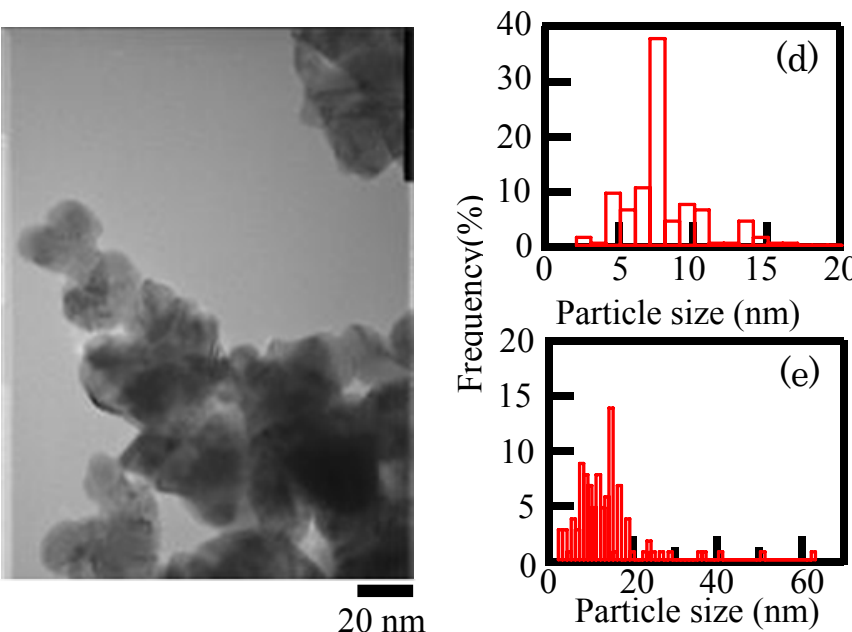

Fig.4 TEM images of the palladium nanoparticles prepared by laser ablation in (a) heavy water, (b) light water and (c) commercial palladium particles (prepared by reduction of palladium salts). Size distribution of palladium nanoparticles prepare by laser ablation in (d) heavy water and (e) light water are shown. The insets in (a) and (b) show electron diffraction pattern.

difference of absorbance of liquild. The most importance for ablation rate is shielding effect of not only used liquid but ablated particles. On the third stage of laser ablation, laser energy can't reach the target surface because of shielding effect of both ablated particles and used liquid. After laser irradiation for 10 minutes, it can be expected to be in the third stage considered from the result shown in Fig. 3 and nanoparticles yield described below. Therefore, ablation should stop after laser irradiation for a few minute because of the interaction of ablated particles and used liquid in this experimental set up. As the result of it, ablation rate should be dominated by laser irradiation for the first few minutes. If the more amount of solvent is used, laser energy might reach onto the target surface for longer time because the increase rate of ablated particles density in liquid is slower. Compared with heavy water and light water, chemical and physical properties such as dipole moment, viscosity which can influence on particle properties $[18,19]$ are almost same. Shielding effect of both ablated particles and used liquid can be expected to influence on nanoparticles yield. In fact, the total yield of ablated nanoparticles in heavy water was $4 \mathrm{mg}$ and that in light water was $3 \mathrm{mg}$. To confirm the detailed influence of absorbance by used liquid, we tried to laser ablation in

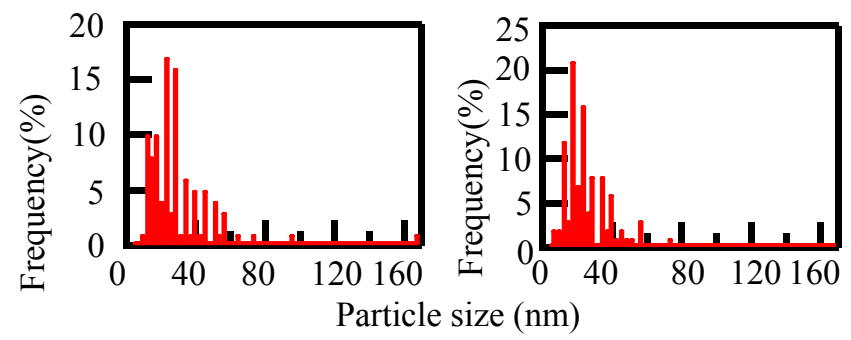

Fig.5 Size distribution of palladium nanoparticles prepared by laser ablation of palladium nanoparticles dispersed in (left) light water or (right) heavy water. mixed liquid of heavy water and light water. However, the difference of the nanoparticls yield is too small to confirm our assumption in this experiment.

\section{3-2 TEM}

The difference between palladium nanoparticles prepared by laser ablation in heavy water and that in light water can be confirmed by TEM images. Fig. 4 shows TEM images of palladium nanoparticles prepared by laser ablation in (a) heavy water, (b) light water and (c) commercial palladium nanoparticles prepared by reduction of palladium salts (99.9\% up KOJUNDO CHEMICAL LABORATORY CO., LTD). The average size of palladium nanoparticles prepared by laser ablation in heavy water or light water were $3,23 \mathrm{~nm}$, respectively. The size distributions shown in Fig.4 (d) and (e) were measured by counting 100 particles. In the case of laser ablation in heavy water, particle size distribution was narrower than that in light

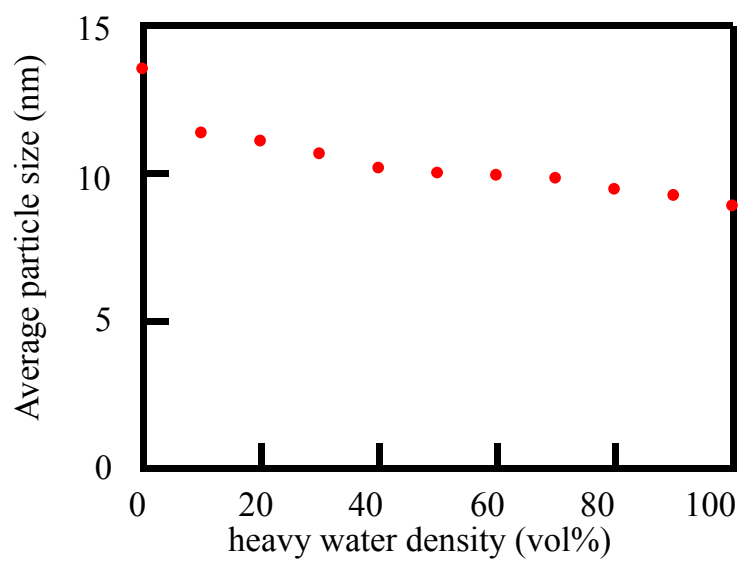

Fig.6 Average size distribution of palladium nanoparticles prepared by laser ablation in mixed liquid of heavy water and light water. 


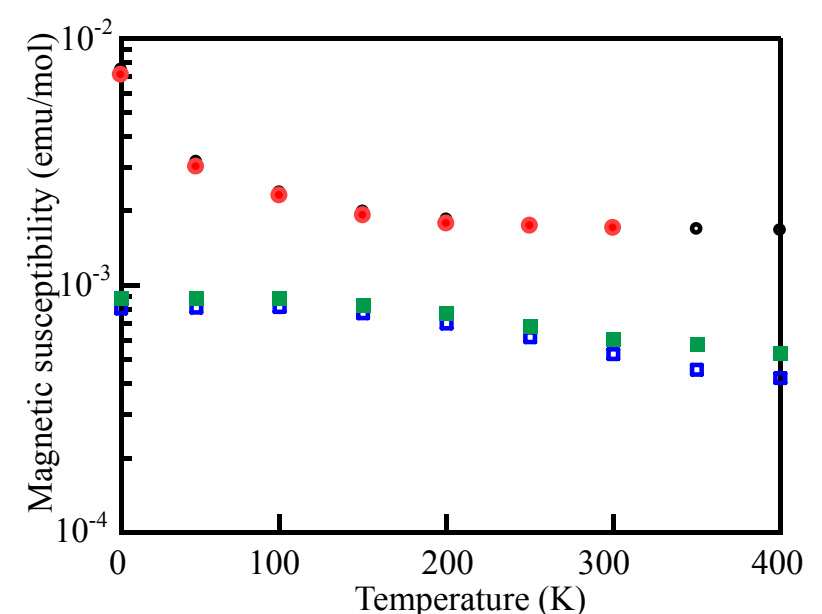

Fig.7 Magnetic susceptibility of palladium nanoparticles prepared by laser ablation in (०) heavy water, (•) light water and commercial palladium ( $\square$ ) powder and ( $\square$ ) foil.

water. As mentioned above, reablation of palladium nanoparticles prepared in heavy water can be expected more easily than that in light water. Therefore, the average size of palladium nanoparticles prepared in heavy water was smaller than that prepared in light water. Influences of many parameters such as flence on nanoparticles properties have been reported. For example, Meunier et al. reported that higher laser fluence produced bigger Au nanoparticles in water[20]. However, Sasaki et al. reported inverse influence in the case of platinum target[10]. These reports may seem to show the contradiction. However, it is difficult to explain the generated particles size distribution by only laser fluence because target properties also effect particle size. In addition, for long time ablation, the influence of reablation of the particles dispersed in liquid decrease the particles size although it can be ignored for short time laser ablation.

To confirm this reablation effect, we carried out experiments of laser ablation of palladium nanoparticles dispersed in heavy water or light water. As the results of them, the size distributions of palladium nanoparticles measured by counting 100 particles in TEM images are shown in Fig.5. Average particle sizes (AV) were $24 \mathrm{~nm}$ and $29 \mathrm{~nm}$ in the case of heavy water and light water, respectively, and standard deviation (SD) were 12 and 20, respectively. This result denotes the difference of the size distributions were smaller than them of palladium nanoparticles prepared by laser ablation of palladium disk target immerged in heavy water or light water. However, it was clear that the size distribution of ablated particles was effected by liquid solvent.

In addition, laser ablation of palladium plate immerged in mixed liquid of heavy water and light water were carried out. Because the dependence of liquid on nanoparticles yield was too small, the detailed dependence of liquid on the nanoparticle yield couldn't be confirmed. The average sizes of palladium nanoparticles measured by counting 100 particles observed by TEM decrease as the concentration of heavy water increases. This result indicates the absorbance of used liquid strongly influences on average

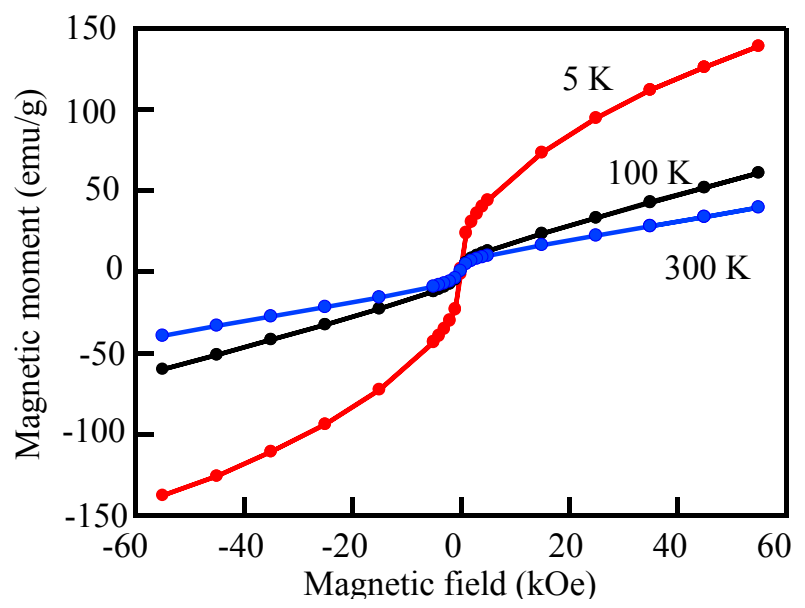

Fig.8 Magnetic moment versus magnetic field curves measured at three different temperature of $5,100,300 \mathrm{~K}$ for palladium nanoparticles produced by laser ablation in heavy water.

particle size. To confirm the dependence of absorbance by liquid, laser ablation of palladium plate in mixed liquid much more times should be carried out.

\section{3-3 Magnetic properties}

Fig.7 shows magnetic susceptibilities of palladium nanoparticles prepared by laser ablation in heavy water, light water and commercial palladium powder and foil with thickness of $25 \mu \mathrm{m}$ (Nilaco). At $5 \mathrm{~K}$, magnetic susceptibilities of palladium nanoparticles prepared by laser ablation in heavy water or light water are ten times as large as commercial palladium powder and foil. To confirm that higher susceptibility is not depended on the oxidation of palladium nanoparticles prepared by laser ablation in liquid, electron diffraction pattern of palladium nanoparticles were obtained and are shown in Fig.4 (a) and (b). While measured magnetic susceptibilities of commercial powder and foil were very similar to other reports of magnetic susceptibility of bulk palladium and that of nanoparticles. And also the susceptibility of ablated particles were more than twice as large as other report[21]. Fig. 8 shows the typical magnetic moment versus magnetic field (M-H) curves taken for palladium nanoparticles prepared by laser ablation in heavy water, providing obvious evidence for superparamagnetism at all measured temperature. The magnetic moment of palladium nanoparticles prepared by laser ablation is more than ten times as large as other report [17]. According to their investigation, magnetic moment increases with the decrease of particle size. However, large magnetic moment can't be explained only by size effect. Reduced coordination number, (in cluster of metal atoms), locally symmetry changes (vacancies and surface state) and electron localization induced by lattice expansion are proposed as the possible key factor that might well induce superparamagnetism in nanoparticles of otherwise nonmagnetic metals [22]. Since ablated particles go through the laser induced plume in which the temperature, 
pressure and electron density are very high, nanoparticles with particular condition which can induce larger magnetic moment may be produced. Hence, nanoparticles produced by laser ablation in liquid can have properties different from that prepared by other methods. To explain our results, the detailed mechanism of laser ablation should be cleared.

\section{Conclusion}

The dependences of absorbance of laser by liquid on properties, yield and size distribution of ablated particles were studied. Pulsed laser ablation of palladium disc target immerged in heavy water or light water were carried out to prepare palladium nanoparticles. Optical absorption of palladium colloids were measured to investigate influence of absorbance of irradiation laser by ablated particles and used liquid. Transmission electron microscopy was used for characterization of average size, size distribution and shape of nanoparticles. Magnetic properties of palladium nanoparticles were measured by Superconducting Quantum Interference Device (SQUID). Both the average size and its standard deviation of palladium nanoparticles prepared by laser ablation in heavy water were smaller than that prepared by laser ablation in light water. After laser ablation for only $10 \mathrm{~min}$, absorption spectrum of palladium colloid prepared in heavy water was larger than that prepared in light water. The yield and average size of nanoparticles are discussed by relating these properties to reablation of nanoparticles induced by previous laser irradiation. After laser ablation for a few minutes, ablated particles and used liquid prevent ablation due to their interaction with laser. Magnetic properties of palladium nanoparticles were different from commercial palladium nanoparticles prepared by reduction of palladium salts. Application of laser ablation to development of new functional materials was shown.

\section{References}

[1]T. Teranishi, and M. Miyake, Chem. Matter, 10, 594 (1998)

[2]T. Li, J. Moon, A. A. Morrone, J. J. Mecholsky, D. R. Talham, J. H. Adair, Langmuir, 15, 4328 (1999)

[3]C. Petit, A. Taleb, M. P. Pileni, Adv. Matter, 10, 259 (1998)

[4]T. Nishi, T. Sakka, H. Oguchi, K. Fukami, Y. H. Ogata, J. Electrochem. Soc, 155, F237 (2008)

[5]M. Z. Xue, Z. W. Fu, Thin Solid Film 516, 8386 (2008)

[6]S. C. Singh, R. Gopal, J. Phys. Chem. C 112, 2812 (2008)

[7]R. M. Tilaki, A. Irajizad, S. M. Mahdavi, Appl. Phys. A 84, 215, (2006)

[8]C. He, T. Sasaki, H. Usui, Y. Shimizu, N. Koshizaki, J. Photochem. Photobiol., A 19166 (2007)

[9]C. B. Hwang, Y. S. Fu, Y. L. Lu, S. W. Jang, P. T. Chou, C. R. C. Wang, S. J. Yu, J. Catal. 195, 336(2000)

[10]W. T. Nichols, T. Sasaki, N. Koshizaki, J. Appl. Phys. 100, 114912 (2006)

[11]T. Tsuji, K. Iryo, Y. Nishimura, M. Tsuji, J. Photochem.
Photobiol. A 145201 (2001)

[12]T. Tsuji, K. Iryo, H. Ohta, and Y. Nishimura, Jpn. J. Appl. Phys, Part2 39, L981 (2000)

[13]T. Okada, J. Suehiro, Appl. Surf. Sci. 253, 7840 (2007)

[14]Y. Kawaguchi, X. Ding, A. Narazaki, T. Sato, H. Niino, Appl. Phys. A79, 883 (2004)

[15]K. Saito, T. Sakka, Y. H. Ogata, J. Appl. Phys. 94, 5530 (2003)

[16]M. J. Zhu, D. M. Bylander, L. Kleinman, Phys. Rev. B 42, 2874 (1990)

[17]S. Angappane, J. Park, Y. Jang, T. Hyeon and J. G. Park, J. Phys. Condensed Matter, 20, 295209 (2008,)

[18]R. M. Tilaki, A. Irajizad, Mahadavi, Appl. Phys. A, 84, 215, 2006

[19]G. Compagnini, A. A. Scalisi, O. Puglisi, J. Appl. Phys. 94, 12, 2003

[20]A. V. Kabashin, M. Meunier et, J. Appl. Phys. 94, 12, 2003

[21]Y. Y. Chen, Y.D. Yao, S. S. Hsiao, S. U. Jen, B. T. Lin, H. M. Lin, and C. Y. Tung, Phys. Rev. B 52, 9364 (1995)

[22]B. Sampedro, P. Crespo, A. Hernando, R. Litrán, J. C. Sánchez-López, C. López-Cartes, A. Fernandez, J.

Ramírez, J. González-Calbet and M. Vallet, Phys. Rev. Lett. 91, 237203 (2003)

(Received: July 13 , 2009, Accepted: June 17, 2010) 